\title{
A monocular ground plane estimation system
}

\author{
D A Castelow and A J Rérolle ${ }^{\dagger}$ \\ GEC Marconi Ltd, Hirst Research Centre, \\ East Lane, Wembley, Middlesex, HA9 7PP, \\ United Kingdom
}

\begin{abstract}
This paper describes the design of a system for estimating the pose of a camera mounted on a vehicle which moves over a flat floor. The system has been designed to work in conjunction with an obstacle avoidance scheme. Measurements are derived from a sequence of images captured by a single camera rigidly mounted on the vehicle and looking at the floor, and combined with measurements of the vehicle position in an extended Kalman filter (EKF). The filter is tested using simulation data.
\end{abstract}

\section{Introduction}

The stereo and monocular obstacle detection and avoidance systems developed by Mallot et al. [1], Sandini et al. [2, 3], and Carlsson and Eklundh [4] all involve offline calibration prior to operation. While prior calibration of any system will always be necessary, on-line recalibration allows for drift or variation in parameters. The filter described here uses data from an AGV and a single CCD camera to solve a constrained structure from motion problem which accomodates variations in camera pose parameters due to vibration or drift. This is similar to a system developed for AGV control [5].

In the following sections we describe the vehicle and camera, introducing the parameters which describe the system (the state variables). The quantities which we measure are described and related to the state variables, the EKF is summarised, and the update equations given. Finally we describe the simulator and some test results.

\section{The Extended Kalman Filter}

State Variables. The position and motion of the camera relative to the fixed ground plane is described by two sets of parameters: the camera pose parameters describe the orientation of the camera with respect to the AGV, and the kinematic parameters describe the movement of the AGV with respect to the ground plane. The Camera Pose and Optics. The pose of the camera on the vehicle is described by a set of transformations between the camera and the vehicle coordinate frames. The parameters used to describe the transformation are indicated in figure 1 .

\footnotetext{
†on industrial placement at HRC from Télécom Paris, 46 rue Barrault, 75634 Paris.
} 

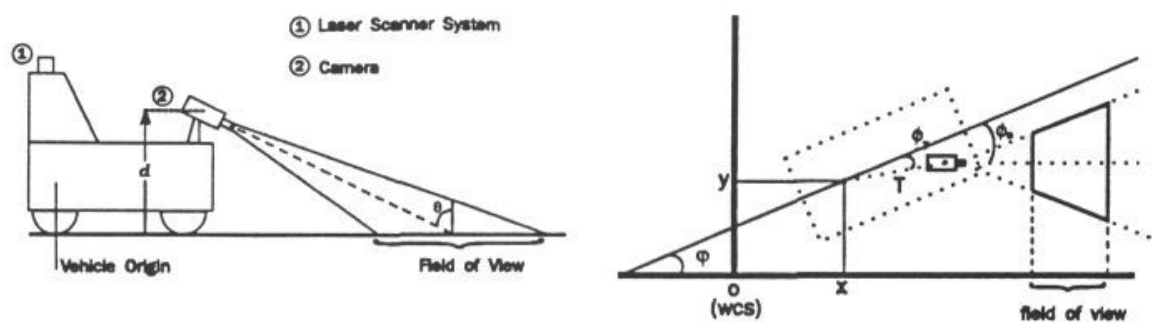

Figure 1: The arrangement of camera and vehicle, side and top views (not to scale).

\begin{tabular}{|c|c|c|c|c|c|c|c|}
\hline \multicolumn{4}{|c|}{ Kinetic parameters } & \multicolumn{4}{|c|}{ Camera Pose parameters } \\
\hline & imeter & Description & Update & & ameter & Description & Update \\
\hline \multicolumn{4}{|c|}{ typical value } & \multicolumn{4}{|c|}{ typical value } \\
\hline$x_{T}$ & & position & $\dot{x_{T}}=u_{T}$ & & $2.2 \mathrm{rad}$ & pitch & $\dot{\theta}=0$ \\
\hline$y_{T}$ & & position & $\dot{y}_{T}=v_{T}$ & & $0.0 \mathrm{rad}$ & roll & $\dot{\psi}=0$ \\
\hline$\varphi$ & & heading & $\dot{\varphi}=\omega_{T}$ & $\phi_{0}$ & $0.1 \mathrm{rad}$ & offset angle & $\dot{\phi}_{0}=0$ \\
\hline$\omega_{T}$ & $0.25 \mathrm{rad} \mathrm{s}^{-1}$ & rotation & $\dot{\omega}_{T}=a_{\omega}$ & $d$ & $500 \mathrm{~mm}$ & height & $\dot{d}=0$ \\
\hline$u_{T}$ & $500 \mathrm{~mm} \mathrm{~s}^{-1}$ & translation & $\dot{u}_{T}=a_{x}$ & $T$ & $1150 \mathrm{~mm}$ & lever & $\dot{T}=0$ \\
\hline$v_{T}$ & $0 \mathrm{~mm} \mathrm{~s}^{-1}$ & translation & $\dot{v}_{T}=a_{y}$ & & & & \\
\hline$a_{\omega}$ & $0 \mathrm{rad} \mathrm{s}^{-2}$ & acceleration & $\dot{a}_{\omega}=0$ & & & & \\
\hline$a_{x}$ & $0.02 \mathrm{~mm} \mathrm{~s}^{-2}$ & acceleration & $\dot{a}_{x}=0$ & & & & \\
\hline$a_{y}$ & $0.02 \mathrm{~mm} \mathrm{~s}^{-2}$ & acceleration & $a_{y}=0$ & & & & \\
\hline
\end{tabular}

Table 1: The state variables and update equations for the ground plane estimator.

In the simulations we assume the images to be $512 \times 512$ with a 768 pixel focal length lens $\left(36^{\circ}\right.$ field of view), and square pixels.

The Vehicle. Our work is based on characteristics of the GEC Electrical Projects AGV [5]. This uses a laser scanner and surveyed barcodes to determine the pose of the AGV in a fixed "world" coordinate system. The associated covariance matrix is also available. In the results presented here, we do not have an accurate model of the vehicle dynamics. Instead we assume that the three components $\left(x_{T}\right.$ and $y_{T}$ position and heading $\left.(\varphi)\right)$ are independent and undergo constant accelerations.

All the parameters are described briefly in table 1.

The Measurement Equation. For instantaneous rotations a plane described by a normal $N=\left(N_{1}, N_{2}, N_{3}\right)$ translating at velocity $\boldsymbol{V}$ and rotating with angular velocity vector $\Omega$ gives rise to a motion field which is quadratic in the image plane (assuming a pin hole camera model).

In a coordinate system with origin at the focal point of the camera the $x$ and $y$ components of the image plane motion field are

$$
\begin{aligned}
& u(x, y)=u_{0}+u_{x} x+u_{x x} x^{2}+u_{y} y+u_{x y} x y \\
& v(x, y)=v_{0}+v_{x} x+v_{y} y+v_{x y} x y+v_{y y} y^{2} .
\end{aligned}
$$

The 12 image distortion parameters (IDP) $\left(u_{0}, u_{x}, \ldots\right)$, (refered to as $\boldsymbol{\beta}$ parameters in $[6$, chapter 6 , pages 105-106]) are related to the components of $\boldsymbol{N}, \boldsymbol{V}, \boldsymbol{\Omega}$, for 


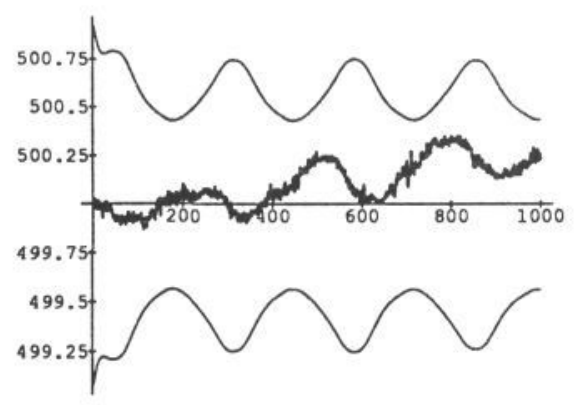

(a)

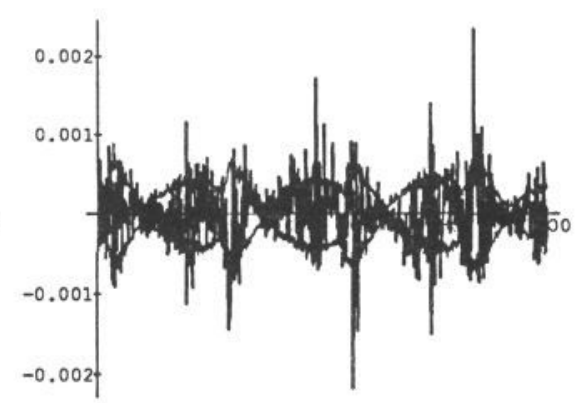

(b)

Figure 2: Filter response with $1 \%$ noise added to motion parameters. (a) estimate of camera height, $d$, and the estimated variance ( \pm 1 std. dev.). (b) the camera roll angle, $\psi$, and the estimated variance ( \pm 1 std. dev.).

example, in a camera centred coordinate frame with coordinate axis 3 along the optic axis,

$$
u_{x}=-\left(N_{1} V_{1}\right)+N_{3} V_{3},
$$

where the components are in a camera centred coordinate frame (coordinate 3 along the optic axis) [6].

The quantities $N, V, \Omega$, may be constructed from the state vectors: e.g. $N_{3}=$ $\frac{1}{d} \cos (\theta)$ where $d$ is the height of the camera and $\theta$ its pitch). The IDP together with the vehicle pose comprise the measurement vector. The measurement equations relate the IDP and the vehicle pose to the state variables. Equation 3 for $u_{x}$ and that for $N_{3}$ and similar are an example. Measurements are obtained by performing a least squares minimisation over a set of visual motion estimates [6, chapter 6]. The visual motion estimates could be determined using, for example, edge matching such as described in $[6$, chapter 2].

The Extended Kalman Filter. The 14 state variables and 15 measured quantities are related by the measurement equation indicated by the equation for $u_{x}$ and similar expressions [7] or [6, chapter 6]. This is used in a continous-discrete EKF (see table 6.1-1 of Gelb [8]) with linear system dynamics which are solved exactly [7].

\section{Simulations}

The EKF described has been tested using simulated data. Plant noise is added to the kinematic state variables to investigate a variety of vehicle trajectories. Measurement noise is added to other state variables, e.g. to $d$ to represent a vibration. The noisy state variables are then used to compute IDPs and these used to generate a set of flow vectors with random orientation, to which measurement noise is added. The flow vectors are used in a least squares minimisation which estimates the IDPs and their covariance matrix. The noise added to the vehicle motion was of order $1-2 \%$, while that added to the states used for generation of the IDPs was of order $0.1 \%$ in each component. The flow vectors were perturbed by approximately $1 \%$. 
Figures 2 shows the response of two components of the state vector (the camera height, $d$ and the camera roll angle, $\psi$ ) when the filter was initialised with the true initial state. The response rate of the filter depends on the assumed uncertainty. The filter showed greater stability to perturbation of some parameters (all kinematic parameters excellent, $\theta$ and $\psi$ very good, $\phi_{0}, d$ and $T$ occasionally poor).

\section{Conclusions}

The design of a system for ground plane estimation has been described, and its implementation tested using simulated data. This has demonstrated that a constrained solution of the structure from motion problem can be used to determine the pose of a camera relative to its supporting vehicle when a vehicle moves across a flat floor. This could provide the basis for a self calibrating obstacle detection scheme using vision techniques such as are described by Carlsson and Eklundh [4] or Sandini and Tistarelli [3].

\section{Acknowledgements}

This research was supported by GEC Marconi Ltd and ESPRIT project 2502, "VOILA". We thank B F Buxton, S J Maybank and C G Harris for encouragement and advice, and J M Davies for bibliographic assistance.

\section{References}

[1] H A Mallot, E Schulze, and K Storjohann. Neural network strategies for robot navigation. In Proc nEuro'88, Paris, June 1988, pages 560-569, 1988.

[2] F Ferrari, E Grosso, M Magrassi, and G Sandini. A stereo vision system for real time obstacle avoidance in unknown envinronment. In Proc of Intl. workshop on Intelligent Robots and Systems, Tokyo, Japan, July 1990. IEEE Comp Soc, 1990.

[3] M Tistarelli and G Sandini. Uncertainty analysis in visual motion and depth estimation from active ego-motion. In SPIE Applications of A.I. VII, volume $1095,1989$.

[4] S Carlsson and J O Eklundh. Object detection using model based prediction and motion parallax. In Proc 1st ECCV, Antibes, France, April 1990, pages 297-306. Springer-Verlag, 1990.

[5] P Stephans. US Patent 4,647,784, 1987.

[6] D W Murray and B F Buxton. Experiments in the Machine Interpretation of Visual Motion. MIT Press, Cambridge, Ma, 1990.

[7] D A Castelow and A J Rérolle. Implementation of an extended Kalman filter for ground plane estimation. Technical report 17,433B, GEC Marconi Ltd, Hirst Research Centre, 1991.

[8] A Gelb, editor. Applied optimal estimation, chapter 6, page 188. MIT Press, Cambridge, Ma, 1974. 